Abstracta Iranica Abstracta Iranica

Revue bibliographique pour le domaine irano-aryen

Volume 40-41 | 2019

Comptes rendus des publications de 2017-2018

\title{
Agnes Korn, Irina Nevskaya (éds). Prospective and Proximative in Turkic, Iranian and beyond
}

\section{Dorian Pastor}

\section{Q OpenEdition}

9 Journals

\section{Édition électronique}

URL : http://journals.openedition.org/abstractairanica/47710

DOI : 10.4000/abstractairanica.47710

ISBN : 1961-960X

ISSN : 1961-960X

Éditeur :

CNRS (UMR 7528 Mondes iraniens et indiens), Éditions de l'IFRI

Référence électronique

Dorian Pastor, « Agnes Korn, Irina Nevskaya (éds). Prospective and Proximative in Turkic, Iranian and beyond », Abstracta Iranica [En ligne], Volume 40-41 | 2019, document 1, mis en ligne le 15 juillet 2019, consulté le 20 avril 2021. URL : http://journals.openedition.org/abstractairanica/47710 ; DOI : https:// doi.org/10.4000/abstractairanica.47710

Ce document a été généré automatiquement le 20 avril 2021.

Tous droits réservés 


\title{
Agnes Korn, Irina Nevskaya (éds). Prospective and Proximative in Turkic, Iranian and beyond
}

\author{
Dorian Pastor
}

\section{RÉFÉRENCE}

Agnes Korn, Irina Nevskaya (eds.). Prospective and Proximative in Turkic, Iranian and beyond. Wiesbaden: Reichert, 2017, 387p. (Iran -Turan 18)

1 L'ouvrage regroupe des articles de divers auteurs spécialisés et a pour objectif la description des prospectifs dans une perspective géographique eurasienne. Cette recherche est principalement basée sur les langues turciques, iraniennes et samoyèdes, ainsi que l'araméen, le mongol, le russe et le khanty.

2 Ayant été identifié récemment, le prospectif est, d'un point de vu typologique, encore assez discuté, ainsi que la dualité prospectif/proximatif. Les premières mentions du prospectif décrit comme une catégorie remontent à Comrie (1976), qui le définit comme étant opposée au parfait, ainsi qu'à Johanson $(1975,1994,2000)$. Cette catégorie, souvent exprimée par des constructions grammaticalisées, dénote un fait actuel lié à une action postérieure comme il est possible de trouver de telles constructions en français avec être sur le point de, mais qui peut aussi être exprimée à travers l'indicatif présent: je pars au travail, dans un contexte où le locuteur n'est toujours pas en mouvement et donc où l'action n'a pas encore commencé à se réaliser.

3 Cette catégorie peu décrite dans la littérature fait donc dans cet ouvrage l'objet d'études dans les langues désignées. Une première partie d'Irina Nevskaya, Agnes Korn et Lars Johanson, fait office de prémisse à cette étude dans une large description qui fait état des discussions en cours sur ce qu'est le prospectif/proximatif, d'une part. D'autre part, une exploration sémantique de ladite catégorie, ainsi qu'une plus large définition de cette dernière. Et enfin, une présentation des possibilités de prospectifs 
dans une perceptive morphologique nous présente des exemples de ce qu'il est possible de trouver dans les langues, en usant notamment des langues archaïques et moyennes de l'iranien. Les deux autres parties de l'ouvrage sont consacrées à l'analyse et l'étude de cas des expressions du prospectif dans les langues turciques, dont le vieux-turc, le turc, l'azéri mais aussi des langues turciques de Sibérie, dans les langues iraniennes, dont le persan, le baloutchi et le kurde. Enfin, les langues samoyèdes, le russe, l'évène, le khanty, le mongol et l'araméen seront également l'objet de cette étude.

4 L'intérêt de cet ouvrage réside dans la redécouverte des formes grammaticalisées des langues observées, en vue d'en identifier les constructions pouvant être décrites comme des prospectifs. Il est vrai que quelques-uns de ces phénomènes ont déjà été décrits par certains auteurs, mais souvent trop brièvement ou de manière trop partielle. Les présentes études s'adonneront donc ici à la tâche de fournir des analyses plus complètes.

\section{AUTEURS}

DORIAN PASTOR

Paris 